Article

\title{
Preliminary Evaluation of the Safety and Probiotic Potential of Akkermansia muciniphila DSM 22959 in Comparison with Lactobacillus rhamnosus GG
}

\author{
Autilia Cozzolino $\left.{ }^{1} \mathbb{(}\right)$, Franca Vergalito ${ }^{1}$, Patrizio Tremonte ${ }^{1}$, Massimo Iorizzo ${ }^{1}(\mathbb{D}$, \\ Silvia J. Lombardi ${ }^{1}$, Elena Sorrentino ${ }^{1, *(\mathbb{D}}$, Delia Luongo ${ }^{2}$, Raffaele Coppola ${ }^{1}$, \\ Roberto Di Marco $^{3}$ (D) and Mariantonietta Succi ${ }^{1}$ \\ 1 Department of Agricultural, Environmental and Food Sciences (DiAAA), University of Molise, \\ Via De Sanctis, 86100 Campobasso, Italy; a.cozzolino@studenti.unimol.it (A.C.); \\ franca.vergalito@unimol.it (F.V.); tremonte@unimol.it (P.T.); iorizzo@unimol.it (M.I.); \\ silvia.lombardi@unimol.it (S.J.L.); coppola@unimol.it (R.C.); succi@unimol.it (M.S.) \\ 2 Institute of Biostructure and Bioimaging of the National Research Council (IBB-CNR), Via Mezzocannone 16, \\ 80134 Napoli, Italy; delia.luongo@unina.it \\ 3 Department of Medicine and Health Sciences "Vincenzo Tiberio", University of Molise, Via De Sanctis, \\ 86100 Campobasso, Italy; roberto.dimarco@unimol.it \\ * Correspondence: sorrentino@unimol.it
}

Received: 22 December 2019; Accepted: 28 January 2020; Published: 30 January 2020

\begin{abstract}
In this study, for the first time, we examined some of the physico-chemical properties of the cell surface of Akkermansia muciniphila DSM 22959, comparing it with those of Lactobacillus rhamnosus GG—one of the most extensively studied probiotic microorganisms. In particular, hydrophobicity, auto-aggregation, co-aggregation, and biofilm formation were investigated. In addition, antibiotic susceptibility, co-culture, and antimicrobial activity of the two strains were compared. Hydrophobicity was evaluated using xylene and toluene, showing that $A$. muciniphila DSM 22959 possessed moderate hydrophobicity. A. muciniphila showed a faster and higher auto-aggregation ability than $\mathrm{Lb}$. rhamnosus GG, but a lower aptitude in biofilm formation. In the co-aggregation test, the best performance was obtained by $L b$. rhamnosus GG. Regarding the susceptibility to antibiotics, the differences between the two strains were remarkable, with $A$. muciniphila DSM 22959 showing resistance to half of the antibiotic tested. Interesting results were also obtained with regard to the stimulating effect of $L b$. rhamnosus GG on the growth of A. muciniphila when co-cultured.
\end{abstract}

Keywords: Akkermansia muciniphila; Lactobacillus rhamnosus GG; hydrophobicity; auto-aggregation; co-aggregation; biofilm formation; co-culture

\section{Introduction}

Akkermansia muciniphila is an oval shaped, gram-negative, strictly anaerobic bacterium belonging to the phylum Verrucomicrobia, and it constitutes 3\% to $5 \%$ of the gut microbial community in both human and other mammalian species [1,2].

It was firstly isolated from a human fecal sample in 2004 [3], and interest in this microorganism increased during time due to its ability to release enzymes into the intestinal tract that help to regulate the balance of mucin, a major component of the mucous layer that resides on the surface of the gastrointestinal mucosa [4]. Human mucin degradation by A. muciniphila provides competitive exclusion against pathogenic mucus degraders and may have a gatekeeping and signaling function [5]. To gain a competitive advantage, $A$. muciniphila has evolved to metabolize the complex glycans provided by the mucus layer as its sole carbon and nitrogen source [6,7]. An efficient colonization 
of A. muciniphila was observed with the highest numbers in the cecum, where most of the mucin is produced [8]. Mucus degradation leads to the production of metabolites, such as acetic acid, propionic acid and oligosaccharides, which may play a role in metabolic health or inflammatory host status [9].

Some authors observed that $A$. muciniphila is abundant in biopsies of healthy subjects and reduced in those of patients with inflammatory bowel disease, obesity, and other diseases [8]. Therefore, due to its low presence in people affected by intestinal disorders and its link with the wellbeing of the mucus layer, A. muciniphila was suggested as a biomarker for the intestinal health [9].

On the other hand, Weir et al. [10] observed that mucin-degrading bacteria, including A. muciniphila, were present in a significantly larger proportion in the feces of colon cancer patients than in healthy subjects. However, they hypothesized that the observed increase in A. muciniphila may be due to an increase of substrate availability as some types of mucin were overexpressed in colon cancers.

Several authors stated that $A$. muciniphila exerts a range of biological activities positively related to human health; it has therefore been widely considered as a novel potential candidate to ameliorate metabolic disorders associated with obesity, diabetes, liver diseases, and cardiometabolic disorders [11]. Indeed, its administration has been shown to profoundly reduce the development of such diseases [12,13].

In addition, this microorganism has been identified in human milk samples immediately after delivery (colostrum), as well as after 1 and 6 months of breastfeeding [14]. Currently, the species is not included by EFSA (European Food Safety Authority) in the QPS (Qualified Presumption of Safety) list [15]. However, all the knowledge acquired so far on this species supports the proposal of A. muciniphila as a next generation probiotic [12].

According to the FAO (Food and Agriculture Organization) and WHO (World Health Organization) [16] recommendations, probiotic strains must meet specific criteria of safety and functionality. For these reasons, this study has been undertaken to preliminary evaluate some features, such as antibiotic susceptibility, physico-chemical properties of bacterial cell surface, biofilm formation and antimicrobial activities, of the human isolated strain A. muciniphila DSM 22959. Studied properties were compared with those of Lactobacillus rhamnosus GG, one of the most widely and extensively studied probiotic strain $[17,18]$ belonging to a species that is generally recognized as safe (GRAS) [19].

\section{Materials and Methods}

\subsection{Bacterial Strains}

The bacterial strains used in this study were Lactobacillus rhamnosus GG, previously isolated from a pharmaceutical preparation (Valio LTD, Helsinki, Finland) as described by Succi et al. [20]; Akkermansia muciniphila DSM 22959; Escherichia coli DSM 5698, and E. coli K12-DH5 (DSMZ, Braunschweig, Germany); Proteus mirabilis ATCC 29906; Enterococcus faecalis ATCC 2912; Staphylococcus aureus ATCC 29213; Lactobacillus acidophilus ATCC 4356 (ATCC-American Type Culture Collection). All the strains were maintained at $-80{ }^{\circ} \mathrm{C}$ in glycerol [21] and propagated twice at $37{ }^{\circ} \mathrm{C}$ in proper media before their use.

\subsection{Antibiotic Susceptibility Test}

The antibiotic susceptibility of Lb. rhamnosus GG and A. muciniphila DSM 22959 was performed with the Etest (Epsilometer test) gradient technology (Biomerieux, Marcy-l'Etoile, France). The antibiotics were selected on the basis of the EFSA document regarding bacteria of human importance and the cut-off values are those indicated in the same document [22].

The Etest strips of chloramphenicol, clindamycin, ampicillin, gentamicin, tetracycline, streptomycin, kanamycin, and erythromycin were used in the concentration range 0.016-256 $\mu \mathrm{g} / \mathrm{mL}$. BHI agar plates (Oxoid Ltd., Hampshire, UK) for A. muciniphila DSM 22959 and LSM agar plates (90\% of iso-sensitest-IST, and $10 \%$ of MRS, Oxoid) for Lb. rhamnosus [23] were inoculated with bacterial suspensions in a sterile saline solution. 
The bacterial cell density of suspensions was adjusted to match McFarland turbidity standard 0.5 using a spectrophotometer (bio-spectrometer basic, Eppendorf, Italy). After drying the surfaces of the plates for 15 to $20 \mathrm{~min}$, Etest strips of tested antibiotics were applied directly onto the surface. The plates were incubated overnight under anaerobic conditions at $37^{\circ} \mathrm{C}$. After the required incubation period, the MIC values were read, detecting the pointed end of the inhibition ellipse that intersects the side of the strip.

\subsection{Hydrophobicity Assay}

The determination of cell surface hydrophobicity was evaluated on Lb. rhamnosus GG and A. muciniphila DSM 22959 based on the bacterial ability to adhere to hydrocarbons. Bacterial adhesion to hydrocarbons (BATH) test was performed using xylene and toluene, according to the procedure described by Collado et al. [24] with some modifications. Briefly, Lb. rhamnosus GG and A. muciniphila DSM 22959 were grown overnight at $37^{\circ} \mathrm{C}$ in MRS broth and BHI broth, respectively. Cells were collected by centrifugation ( $8000 \mathrm{rpm}$ for $10 \mathrm{~min}$ at $4{ }^{\circ} \mathrm{C}$ ), washed twice and re-suspended in a sterile phosphate buffer saline (PBS, pH 7) to achieve an optical density (OD $580 \mathrm{~nm}$ ) of $0.5( \pm 0.05)$, in order to standardize the number of bacteria (7-8 Log CFU/mL). Then, an equal volume of hydrocarbon (xylene or toluene) was added. The two-phase system was thoroughly mixed by vortexing for 5 min. The aqueous phase was carefully removed after 15, 30, and $60 \mathrm{~min}$ of incubation at room temperature and its absorbance at $580 \mathrm{~nm}$ was measured using a spectrophotometer. Affinity to hydrocarbons (hydrophobicity) was calculated using the following formula:

$$
\text { Hydrophobicity } \%=\left(\frac{O D_{0}-O D_{\text {final }}}{O D_{0}}\right) \times 100 \text {, }
$$

where $\mathrm{OD}_{0}$ and $\mathrm{OD}_{\text {final }}$ are the absorbance values before and after extraction with hydrocarbons, respectively.

Hydrophobicity was calculated as the percentage decrease in the optical density of the initial bacterial suspension, as the hydrophilic bacteria will be located in the water, while the hydrophobic bacteria will be associated with hydrocarbons [24].

\subsection{Auto-Aggregation Assay}

The auto-aggregation assay was performed as described by Collado et al. [24]. Briefly, cell suspensions of $L b$. rhamnosus GG and A. muciniphila DSM 22959 were prepared as described above and incubated at $37^{\circ} \mathrm{C}$. OD was adjusted to $0.5( \pm 0.05)$. Then the bacterial suspensions were incubated at $37^{\circ} \mathrm{C}$ and their OD were detected at 1, 2, 5, and $24 \mathrm{~h}$.

Auto-aggregation percentage was calculated using the following formula [25]:

$$
\text { Auto-aggregation } \%=1-\left(\frac{O D_{\text {final }}}{O D_{0}}\right) \times 100 \text {, }
$$

where $\mathrm{OD}_{0}$ is the absorbance at time 0 , and $\mathrm{OD}_{\text {final }}$ is the absorbance detected after $1,2,5$, and $24 \mathrm{~h}$.

\subsection{Biofilm Formation}

Biofilm formation was evaluated on Lb. rhamnosus GG and A. muciniphila DSM 22959 under static conditions, as described by Stepanović et al. [26] with some modifications. Strains were grown overnight at $37^{\circ} \mathrm{C}$ in tryptic soy broth (TSB, Oxoid). Then, bacterial cells were harvested by centrifugation at $8000 \mathrm{rpm}$ for $10 \mathrm{~min}$ at $4{ }^{\circ} \mathrm{C}$, washed twice with PBS and resuspended in the following media: TSB without glucose and TSB supplemented with $0.25 \%, 1 \%$, and $2.5 \%$ D-glucose. Three aliquots of $200 \mu \mathrm{L}$ of each bacterial suspension were filled in a 96-well polystyrene microtiter plate. Negative controls were constituted by wells filled with uninoculated culture media. Microtiter plates were incubated for $24 \mathrm{~h}$ at $37^{\circ} \mathrm{C}$. The medium was removed by each well and plates were washed three 
times with a sterile saline solution to remove unattached cells. The remaining attached cells were fixed with $200 \mu \mathrm{L}$ of $99 \%$ methanol (Sigma-Aldrich) per well. After $15 \mathrm{~min}$, wells were emptied and left to dry. Then wells were stained for $5 \mathrm{~min}$ with $200 \mu \mathrm{L}$ of $2 \%$ Crystal Violet (Liofilchem, Italy) per well. Excess stain was removed by washing three times with sterile saline solution. After the plates were air-dried, the adherent cells were resuspended in $160 \mu \mathrm{L}$ of $33 \%(\mathrm{v} / \mathrm{v})$ glacial acetic acid (Sigma-Aldrich). The OD of each well was measured at $580 \mathrm{~nm}$ by using an automated PerkinElmer 1420 Multilabel Counter.

The cut-off $\left(\mathrm{OD}_{\mathrm{C}}\right)$ was defined as the mean $\mathrm{OD}$ value of the negative control. Based on the $\mathrm{OD}$, strains were classified as non- biofilm producers $\left(\mathrm{OD} \leq \mathrm{OD}_{\mathrm{C}}\right)$, weak $\left(\mathrm{OD}_{\mathrm{C}}<\mathrm{OD} \leq 2 \times \mathrm{OD}_{\mathrm{C}}\right)$, moderate $\left(2 \times \mathrm{OD}_{\mathrm{C}}<\mathrm{OD} \leq 4 \times \mathrm{OD}_{\mathrm{C}}\right)$, or strong biofilm producers $\left(4 \times \mathrm{OD}_{\mathrm{C}}<\mathrm{OD}\right)$.

\subsection{Co-Culture of Lb. rhamnosus and A. muciniphila}

This test was performed following the protocol described by Ruiz et al. [27] with some modifications. Strains were grown at $37^{\circ} \mathrm{C}$ in BHI broth, cells were collected by centrifugation ( $8000 \mathrm{rpm}$ for $10 \mathrm{~min}$ at $4{ }^{\circ} \mathrm{C}$ ) from the early logarithmic growth phase, washed twice, and re-suspended in sterile saline solution to an optical density $(580 \mathrm{~nm}$ ) of about 0.5 . The cell suspensions were individually (only Lb. rhamnosus GG and only A. muciniphila DSM 22959) inoculated (1\% v/v) in tubes of BHI broth preheated at $37^{\circ} \mathrm{C}$, and incubated at $37^{\circ} \mathrm{C}$. The same procedure was used on the strains in co-culture. In this last case, the co-culture tubes were vigorously vortexed before incubation in order to promote the mix between the two bacteria. At time 0 , and after 3, 6, and $24 \mathrm{~h}$ of incubation, microbial counts were performed on single cultures $L b$. rhamnosus GG and A. muciniphila DSM 22959, and on the co-culture. For co-culture, $\mathrm{Lb}$. rhamnosus GG was counted in MRS agar added with kanamycin at $37^{\circ} \mathrm{C}$ in anaerobiosis. A. muciniphila DSM 22959 was counted on BHI agar added with chloramphenicol at $37^{\circ} \mathrm{C}$ in anaerobiosis.

\subsection{Co-aggregation Assay}

The ability of $L b$. rhamnosus GG and A. muciniphila DSM 22959 to co-aggregate with E. coli DSM 5698, E. coli K12-DH5, P. mirabilis ATCC 29906, Ec. faecalis ATCC 2912, S. aureus ATCC 29213, and Lb. acidophilus ATCC 4356 was evaluated following the procedure reported by Collado et al. [24].

Bacterial suspensions were prepared as described for BATH test. Equal volumes ( $500 \mu \mathrm{L})$ of Lb. rhamnosus GG or A. muciniphila DSM 22959 were mixed with each of the other strains for $10 \mathrm{~s}$ on a vortex and incubated at $37^{\circ} \mathrm{C}$ without agitation.

Absorbance $(580 \mathrm{~nm}$ ) of each mix, and of each bacterial suspension (controls) was monitored at 5 and $24 \mathrm{~h}$ of incubation. The percentage of co-aggregation was calculated using the following formula [28]:

$$
\text { Co- aggregation } \%=\left(\frac{\left[\left(O D_{x}+O D_{y}\right) / 2\right]-O D_{m i x}}{\left(O D_{x}+O D_{y}\right) / 2}\right) \times 100,
$$

where $\mathrm{OD}_{\mathrm{x}}$ and $\mathrm{OD}_{\mathrm{y}}$ represent the absorbance values of the separate bacterial suspensions in control tubes, and $\mathrm{OD}_{\text {mix }}$ represent the absorbance values of the mixed bacterial suspensions at 5 and $24 \mathrm{~h}$.

\subsection{Antimicrobial Activity}

The antimicrobial activity of Lb. rhamnosus GG and A. muciniphila DSM 22959 (producers) was evaluated against E. coli DSM 5698, E. coli K12-DH5, P. mirabilis ATCC 29906, Ec. faecalis ATCC 2912, S. aureus ATCC 29213 and Lb. acidophilus ATCC 4356 (indicators).

The antimicrobial activity of the strains was conducted according to the agar well diffusion assay described by Tremonte et al. [29] with same modifications. Briefly, $20 \mathrm{~mL}$ of BHI soft agar $(0.7 \%$ agar $)$ inoculated with an overnight culture of each indicator strain (final concentration of about 7 Log $\mathrm{CFU} / \mathrm{mL}$ ) were poured in Petri plates. Wells of $5.0 \mathrm{~mm}$ in diameter were bored into plates and $50 \mu \mathrm{L}$ of each producer strain was placed into each well. After incubation at $37^{\circ} \mathrm{C}$ for 24 to $48 \mathrm{~h}$, the plates 
were observed for the inhibition zones and the inhibition halos were normalized using the following formula [30]:

$$
\text { Inhibition score (IS) }=\varnothing \text { inhibition halo }(\mathrm{mm}) / \varnothing \text { well }(\mathrm{mm}) \text {. }
$$

On this basis, the antimicrobial effect was considered as low $(1<$ IS $<3)$, moderate $(3 \leq$ IS $<5)$, strong $(5 \leq$ IS $<7$ ) or very strong $(7 \leq$ IS $<9)$.

A calibrated-densitometer (GS-800, Bio-Rad, Hermles CA, USA) was used for imaging acquisition and Adobe Photoshop CS4 Extended software was used for the measurement of clearing zones.

\subsection{Statistical Analysis}

All data were expressed as mean \pm standard deviation (SD) of the measurements were carried out in duplicate on three independent experiments. Statistical analysis was performed through the analysis of variance (ANOVA) followed by the Tuckey's multiple comparison. Statistical significance was attributed to $p$ values $<0.05$. The software SPSS (IBM SPSS Statistics 21) was used for the analysis.

\section{Results and Discussion}

This study aimed at the preliminary evaluation of some probiotic features of the human isolated strain A. muciniphila DSM 22959. The strain Lb. rhamnosus GG was chosen for comparative purposes, considering that it is one of the most extensively studied and well characterized probiotic strains [31-34]. In addition, $L b$. rhamnosus GG is widely consumed worldwide and it is also used in many pharmaceutical formulas and fermented milk-based preparations $[17,18]$.

\subsection{Antimicrobial Susceptibility}

Antibiotic response is a very important feature for selecting probiotic strains. In fact, probiotics should have low antibiotic resistance and should not possess transmissible antibiotic resistance that can lead to the development of new antibiotic-resistant pathogens [35,36].

For this reason, in this study we tested the susceptibility of A. muciniphila DSM 22959 to several antibiotics (chloramphenicol, ampicillin, clindamycin, tetracycline, gentamicin, streptomycin, kanamycin, and erythromycin) selected on the basis of the EFSA document regarding bacteria of human importance [22].

The results are shown in Table 1. As expected, the differences between $L b$. rhamnosus GG and A. muciniphila DSM 22959 were remarkable for the majority of the tested antibiotics, since Lb. rhamnosus GG and A. muciniphila are Gram positive and Gram negative bacteria, respectively.

The antibiotic susceptibility of $L b$. rhamnosus GG was already reported in a previous study [35], and it was repeated only for comparative purposes. In detail, results confirmed the susceptibility of the probiotic strain $L b$. rhamnosus GG to tested antibiotics, with the exception of kanamycin.

Instead, A. muciniphila was resistant to half of the tested antibiotics, i.e., to chloramphenicol, clindamycin, streptomycin and erythromycin. To the best of our knowledge, only Dubourg et al. [37] assessed the resistance of $A$. muciniphila to different antibiotics, but they tested antimicrobial substances different from those used in the present study. Moreover, Guo et al. [38] observed the acquisition of antibiotic resistance genes during $A$. muciniphila evolution from other species present in the human intestine, induced by the high levels of antibiotics in this environment.

However, in the same field, Cani et al. [12] reported that the inspection of the genome sequence of A. muciniphila did not reveal antibiotic resistance genes that are linked to known genetically transferrable elements. 
Table 1. Antimicrobial susceptibility of A. muciniphila DSM 22959 and Lb. rhamnosus GG against chloramphenicol (CHL), ampicillin (AMP), clindamycin (CLI), tetracycline (TET), gentamicin (GEN), streptomycin (STR), kanamycin (KAN), and erythromycin (ERY), as determined with Etest. MIC values were expressed as $\mu \mathrm{g} / \mathrm{mL}$.

\begin{tabular}{lcccccccc}
\hline \multicolumn{1}{c}{ Strains } & CHL & AMP & CLI & TET & GEN & STR & KAN & ERY \\
\hline A. muciniphila DSM 22959 & 256 & 2 & 256 & 0.75 & 4 & 128 & 12 & 64 \\
Lb. rhamnosus GG & 3 & 1.5 & 0.5 & 0.5 & 8 & 24 & 256 & 0.064 \\
\hline \multicolumn{1}{c}{ Cut-off values [22] } & $\mathbf{4}$ & $\mathbf{4}$ & $\mathbf{1}$ & $\mathbf{8}$ & $\mathbf{1 6}$ & $\mathbf{3 2}$ & $\mathbf{6 4}$ & $\mathbf{1}$ \\
\hline \multicolumn{2}{c}{ Resistant; } & Susceptible. & & &
\end{tabular}

\subsection{Hydrophobicity}

The adhesion ability of bacteria plays an important role in the intestinal colonization. Therefore, this property has been considered as a potential probiotic marker along with other desirable attributes used for the selection of novel probiotic strains [39]. As it is difficult to investigate bacterial adhesion in vivo, an interest has been drawn in the development of in vitro models for preliminary screening of potentially adherent strains. For this reason, effective methods for controlling microbial adhesion have been studied [40]. In particular, the physical and chemical properties of the bacterial cell surface as the hydrophobicity and the auto-aggregation ability, could give some information on the ability of a strain to interact with its environment [41,42]. Specifically, the hydrophobicity is most likely due to complex interactions between positive and negative charges, between hydrophobic and hydrophilic components characterizing the bacterial surface $[24,25,43]$.

The BATH test has been extensively used for evaluating cell surface hydrophobicity in lactic acid bacteria $[24,28,43]$.

Studies on the microbial cell surface have shown that the presence of (glycol-) proteinaceous material results in higher hydrophobicity, whereas hydrophilic surfaces are associated with the presence of polysaccharides [24,29]. Hydrophobic cell surfaces were demonstrated to be highly adherent-more than $40 \%$-to apolar solvents $[24,43]$.

Moreover, Collado et al. [24] have found that hydrophobicity is related to auto-aggregation properties, since probiotic strains with high adhesion in the presence of hydrocarbons have shown high capability of auto-aggregation.

Starting from these considerations, in the present study the hydrophobicity of tested strains was evaluated using xylene and toluene, and the results are reported in Table 2.

Table 2. Adhesion of A. muciniphila DSM 22959 and $L b$. rhamnosus GG to hydrocarbons (expressed as hydrophobicity \%) measured using the BATH test after 15, 30, and $60 \mathrm{~min}$ (contact time).

\begin{tabular}{cccc}
\hline Strains & Contact Time (min) & Xylene & Toluene \\
\hline & 15 & $6.9( \pm 0.5)$ & $25.6( \pm 0.4)$ \\
A. muciniphila DSM 22959 & 30 & $38.0( \pm 0.6)$ & $40.7( \pm 0.6)$ \\
& 60 & $39.4( \pm 0.3)$ & $43.7( \pm 0.4)$ \\
\hline \multirow{2}{*}{ Lb. rhamnosus GG } & 15 & $55.9( \pm 0.3)$ & $48.2( \pm 0.4)$ \\
& 30 & $64.0( \pm 0.6)$ & $48.5( \pm 0.3)$ \\
& 60 & $64.0( \pm 0.4)$ & $49.4( \pm 0.6)$ \\
\hline
\end{tabular}

The strains showed different hydrophobic features. In particular, A. muciniphila DSM 22959 showed lower levels of hydrophobicity compared to Lb. rhamnosus GG. Furthermore, Lb. rhamnosus GG showed higher levels of adhesion to xylene compared to toluene. On the other hand, A. muciniphila DSM 22959 showed slightly higher adhesion to toluene.

The results obtained for A. muciniphila DSM 22959 are not comparable with other studies, due to the lack of information. On the other hand, data regarding Lb. rhamnosus GG are partially confirmed by those reported in the literature. Collado et al. [24] and Tuo et al. [44] observed a hydrophobicity of 
Lb. rhamnosus GG with xylene similar to that recorded in this study (64\% after $60 \mathrm{~min}$ ), while Xu et al. [45] found values of hydrophobicity in the presence of xylene slightly lower than $50 \%$.

Lactobacilli could be classified into three groups: those with low ( $0 \%$ to $35 \%$ ), moderate ( $36 \%$ to $70 \%)$, and high hydrophobicity (71\% to $100 \%)$ [46]. Based on these ranges, and arbitrarily using them for A. muciniphila as well, our results indicate that Lb. rhamnosus GG and A. muciniphila DSM 22959 had a moderate hydrophobicity.

\subsection{Auto-Aggregation}

In order to exert beneficial effects, probiotics need to achieve an adequate mass through aggregation. Consequently, the ability of probiotics to aggregate is considered a desirable property [47]. In fact, auto-aggregation ability of probiotics seems to have influence on their adhesion to intestinal epithelial cells $[47,48]$. The auto-aggregation ability of tested strains was determined at different times $(1,2$, 5 , and $24 \mathrm{~h}$ ). In general, the auto-aggregation ability increased over time in accordance with results obtained by other authors [24,49] (Table 3).

Table 3. Auto-aggregation percentages of A. muciniphila DSM 22959 and Lb. rhamnosus GG strains evaluated after $1,2,5$, and $24 \mathrm{~h}$ of incubation.

\begin{tabular}{ccccc}
\hline & \multicolumn{5}{c}{ \% Auto-Aggregation } \\
\hline Strains & $\mathbf{1 ~ h}$ & $\mathbf{2 ~ h}$ & $\mathbf{5 ~ h}$ & $\mathbf{2 4} \mathbf{~ h}$ \\
\hline A. muciniphila DSM 22959 & $3.59 \pm 0.29$ & $28.70 \pm 1.38$ & $30.94 \pm 2.36$ & $69.42 \pm 4.51$ \\
Lb. rhamnosus GG & $4.67 \pm 0.12$ & $15.19 \pm 0.73$ & $20.13 \pm 1.0$ & $65.21 \pm 2.9$ \\
\hline
\end{tabular}

In particular, after $24 \mathrm{~h}, \mathrm{Lb}$. rhamnosus GG showed a percentage of aggregation of $65.21 \%$-very similar to that observed by Collado et al. [24]. A. muciniphila DSM 22959 showed not only a slightly higher percentage of aggregation at $24 \mathrm{~h}(69.42 \%)$, but also a faster auto-aggregation ability. In fact, after $2 \mathrm{~h}$, it reached a percentage of $28.7 \%$ compared to that of $15.19 \%$ showed by Lb. rhamnosus GG.

Tuo et al. [44] reported a significant correlation $(p<0.01)$ between auto-aggregation and hydrophobicity of some Lactobacillus strains. Our results confirmed the hypothesis that the BATH test is related to auto-aggregation properties only for $L b$. rhamnosus GG, but not for $A$. muciniphila. For this strain, no correlation between hydrophobicity and auto-aggregation was observed (data not shown).

\subsection{Biofilm Formation}

The experiments performed in this study allowed to measure the rate of adherence and subsequent biofilm formation of tested bacteria.

Lb. rhamnosus GG, as reported by some authors, does not form biofilms in MRS; therefore, this medium was omitted from the experiment [50] and TSB was used [26].

In order to study the influence of glucose on biofilm formation, TSB without glucose or with different concentrations of this sugar was used.

The results, summarized in Table 4, showed that $L b$. rhamnosus GG was able to form biofilms inall conditions. In detail, it was strongly adherent (SA), i.e., capable of forming biofilms, with $0 \%$, $0.25 \%$, and $1.0 \%$ of glucose, while, it was moderately adherent (MA) at a concentration of $2.5 \%$ of glucose. These results showed that a high presence of glucose caused a reduction of biofilm formation in Lb. rhamnosus GG.

On the other hand, A. muciniphila DSM 22959 was classified as weakly adherent (WA) in all tested cases, regardless of the glucose concentration used. 
Table 4. Biofilm formation of A. muciniphila DSM 22959 and $L b$. rhamnosus GG after incubation at $37^{\circ} \mathrm{C}$ for $24 \mathrm{~h}$ in TSB containing different concentrations of glucose $(0 \%, 0.25 \%, 1 \%$, and $2.5 \%)$. The cut-off $\left(O D_{C}\right)$ was defined as the mean $\mathrm{OD}$ value of the negative control. Based on the $\mathrm{OD}$, strains were classified as not adherent $\left(\mathrm{OD} \leq \mathrm{OD}_{\mathrm{C}}\right)$, weakly adherent $\left(\mathrm{OD}_{\mathrm{C}}<\mathrm{OD} \leq 2 \times \mathrm{OD}_{\mathrm{C}}\right)$, moderately adherent $\left(2 \times \mathrm{OD}_{\mathrm{C}}<\mathrm{OD} \leq 4 \times \mathrm{OD}_{\mathrm{C}}\right)$ or strongly adherent $\left(4 \times \mathrm{OD}_{\mathrm{C}}<\mathrm{OD}\right)$.

\begin{tabular}{ccccc}
\hline Strains & TSB 0\% & TSB 0.25\% & TSB 1\% & TSB 2.5\% \\
\hline A. muciniphila DSM 22959 & WA & WA & WA & WA \\
Lb. rhamnosus GG & SA & SA & SA & MA \\
\hline
\end{tabular}

$\mathrm{SA}$ = strongly adherent; $\mathrm{MA}$ = moderately adherent; WA = weakly adherent.

\subsection{Co-Culture of A. muciniphila DSM 22959 and Lb. rhamnosus GG}

Co-culture is an experimental model of rather simple culture, which allows the study of interactions between different species, likewise to what would happen in vivo, where the different microorganisms interact with each other, exchanging chemical signals, metabolites, etc. [51].

In this test, an in vitro model was set up to evaluate the interactions between A. muciniphila DSM 22959 and Lb. rhamnosus GG. The purpose of this test was to determine whether the two strains were negatively or positively influenced by each other or were not affected at all. Individual cultures of A. muciniphila DSM 22959 and Lb. rhamnosus GG were used as controls.

On the basis of the results obtained by the antimicrobial susceptibility test, kanamycin and chloramphenicol were selected to count A. muciniphila DSM 22959 and Lb. rhamnosus GG in co-culture. In fact, A. muciniphila DSM 22959 showed a high sensitivity to kanamycin while Lb. rhamnosus GG was resistant to this antibiotic. In the same manner, $L b$. rhamnosus GG was susceptible to chloramphenicol, while A. muciniphila DSM 22959 was resistant.

Figure 1 shows the results obtained by the growth in co-culture. For A. muciniphila DSM 22959 significant differences were observed if it grew alone or in co-culture with Lb. rhamnosus GG. In the first hours A. muciniphila grew faster alone than in co-culture, and this difference was particularly evident at $6 \mathrm{~h}$. Subsequently, the situation completely changed and at $24 \mathrm{~h}$, A. muciniphila DSM 22959 alone reached $8.4 \mathrm{Log} \mathrm{CFU} / \mathrm{mL}$, while in co-culture it reached a value of $9.6 \mathrm{Log} \mathrm{CFU} / \mathrm{mL}$. Therefore, even if the growth of A. muciniphila in co-culture was initially slower, it was favored by the presence of Lb. rhamnosus GG during time.

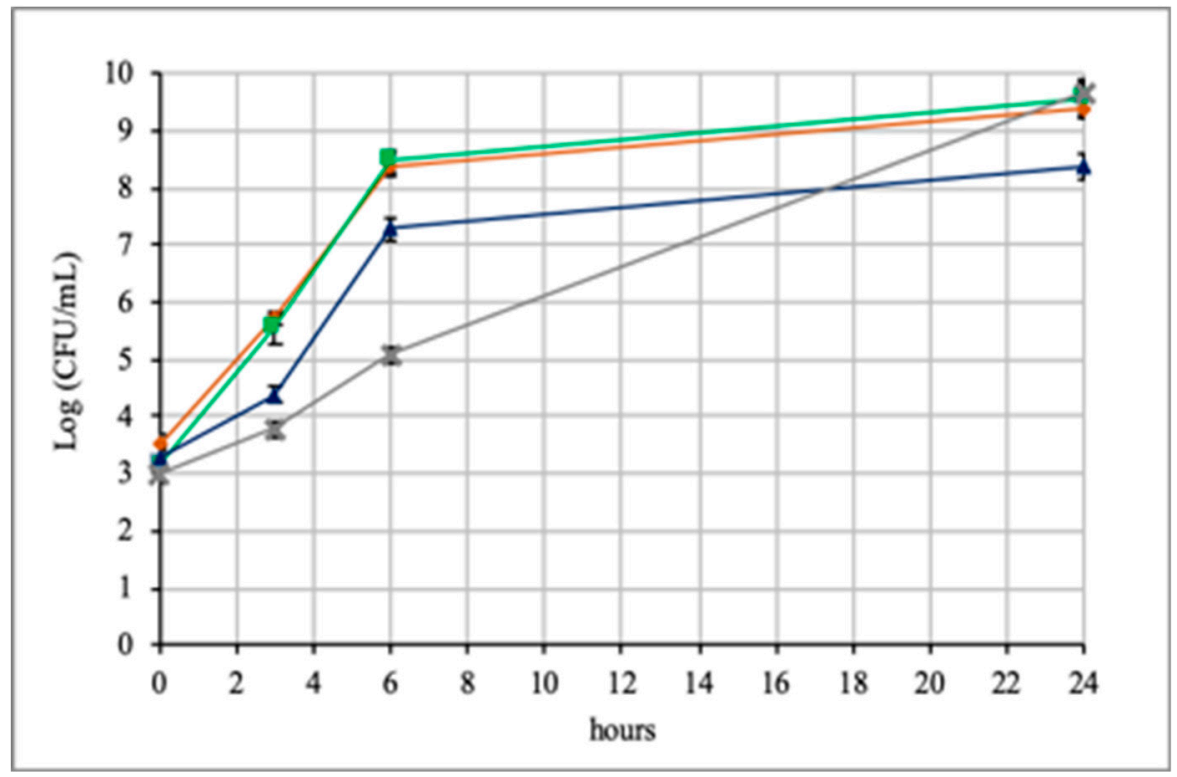

Figure 1. Growth of A. muciniphila DSM 22959 and Lb. rhamnosus GG in co-culture $($ A. muciniphila = $\mathbf{\Delta}$; Lb. rhamnosus $=\varpi ; A$. muciniphila in co-culture $=\mathrm{X} ;$ Lb. rhamnosus in co-culture $\diamond)$. 
Contrarily to previous results, no differences were observed for $L b$. rhamnosus GG. In fact, for this strain the value reached after $24 \mathrm{~h}$ in co-culture $(9.5 \mathrm{Log} \mathrm{CFU} / \mathrm{mL})$ was very similar to that of Lb. rhamnosus GG cultured alone (9.6 Log CFU/mL). Therefore, Lb. rhamnosus GG was not influenced by the presence of $A$. muciniphila DSM 22959.

Based on these results, it is possible to state that $L b$. rhamnosus GG positively influenced the growth of A. muciniphila in vitro. In fact, after $24 \mathrm{~h} \mathrm{~A}$. muciniphila in co-culture reached a value of 1.2 Log higher than that reached alone.

The ability of $L b$. rhamnosus GG to stimulate the growth of $A$. muciniphila DSM 22959 is an interesting result, considering that $L b$. rhamnosus GG is widely used in probiotic preparations. However, this positive influence will have to be confirmed in vivo, where many other variables are involved.

\subsection{Co-aggregation}

Co-aggregation is an important feature for probiotics, because it may play a crucial role in the prevention of colonization of gastrointestinal tract (GIT) by pathogens [48]. In fact, probiotic strains can form a barrier that prevents colonization by pathogens through co-aggregation [52]. Moreover, co-aggregation with a potential pathogen allows probiotics to produce antimicrobial substances in very close proximity to them, which may inhibit the growth of pathogenic strains in the GIT [44].

In this study, we examined the ability of Lb. rhamnosus GG and A. muciniphila DSM 22959 to co-aggregate with some commensal/pathogen bacteria such as E. coli, Proteus mirabilis, Enterococcus faecalis, Staphylococcus aureus, and Lb. acidophilus. The results of this test are shown in Table 5.

Table 5. Co-aggregation percentages of Lb. rhamnosus GG and A. muciniphila DSM 22959 with E. coli DSM 5698, E. coli K12-DH5, P. mirabilis ATCC 29906, Ec. faecalis ATCC 2912, S. aureus ATCC 29213, and $L b$. acidophilus ATCC 4356 evaluated after 5 and $24 \mathrm{~h}$ of incubation.

\begin{tabular}{ccccc}
\hline & \multicolumn{5}{c}{ Co-Aggregation } \\
\hline Strains & \multicolumn{2}{c}{ Lb. rhamnosus GG } & \multicolumn{2}{c}{ A. muciniphila DSM 22959 } \\
& $\mathbf{5 ~ h}$ & $\mathbf{2 4} \mathbf{~ h}$ & $\mathbf{5 ~ h}$ & $\mathbf{2 4} \mathbf{~ h}$ \\
\hline Ec. faecalis ATCC2912 & $16.39 \pm 1.8$ & $40.64 \pm 3.6$ & $23.81 \pm 2.2$ & $44.31 \pm 4.0$ \\
P. mirabilis ATCC $\mathbf{2 9 9 0 6}$ & $26.20 \pm 1.9$ & $66.86 \pm 3.1$ & $24.51 \pm 2.3$ & $47.46 \pm 3.9$ \\
S. aureus ATCC 29213 & $8.29 \pm 0.7$ & $39.23 \pm 1.3$ & $20.64 \pm 3.3$ & $40.48 \pm 3.6$ \\
E. coli DSM 5698 & $35.70 \pm 8.1$ & $60.40 \pm 2.9$ & $2.67 \pm 0.11$ & $6.15 \pm 0.25$ \\
E. coli K12-DH5 & $34.80 \pm 3.5$ & $58.30 \pm 2.25$ & $2.31 \pm 0.21$ & $6.10 \pm 0.30$ \\
Lb. acidophilus ATCC 4356 & $40.06 \pm 3.5$ & $72.26 \pm 6.1$ & $24.77 \pm 3.6$ & $54.38 \pm 4.2$ \\
\hline
\end{tabular}

In general, Lb. rhamnosus GG showed co-aggregation abilities with all bacteria tested, but with different percentages. After $24 \mathrm{~h}$ of incubation, Lb. rhamnosus GG showed high ability to co-aggregate with P. mirabilis ATCC 29906 (66.86\%), E. coli DSM 5698 (60.40\%) and E. coli K12-DH5 (58.30\%), in agreement with Collado et al. [24]. Moreover, Lb. rhamnosus GG showed the highest ability to co-aggregate with $\mathrm{Lb}$. acidophilus (72.26\%).

A. muciniphila showed a good ability of co-aggregation with Ec. faecalis ATCC 2912 (44.31\%), and S. aureus ATCC 29213 (40.48\%), comparable to that observed for GG, and with P. mirabilis ATCC 29906 (47.46\%). Moreover A. muciniphila also showed high co-aggregation percentages with Lb. acidophilus ATCC 4356.

According to other authors [46], the co-aggregation percentage was found to depend on the contact time.

Several authors suggest that the ability to co-aggregate with pathogens can be used for the preliminary selection of probiotic strains $[24,52,53]$, considering that co-aggregation was demonstrated to be strain specific. 


\subsection{Antimicrobial Activity}

Human gastrointestinal tract is colonized by a vast community of commensal microorganisms that have important effects on immune function, nutrient processing, and a broad range of other host activities. These microorganisms can influence each other positively or negatively. The interactions that are established among the components of the microbiota can strongly affect the human health. For these reasons, it is important to know the interaction that occur between probiotics and commensal and/or pathogen microorganisms. In this field, we examined the interactions between Lb. rhamnosus GG, A. muciniphila DSM 22959, and the same commensal/pathogen bacteria used for the co-aggregation assay. The agar well diffusion technique was used to detect if the growing cells of $L b$. rhamnosus GG and A. muciniphila DSM 22959 (producers), had inhibitory properties against the indicator strains.

For A. muciniphila DSM 22959 no halos of inhibition were found, evidencing that it was unable to inhibit all indicators (data not shown). Similar results were observed for Lb. rhamnosus GG, which exerted a moderate antimicrobial activity only against $E$. coli DSM5698 (diameter of inhibition halo $2.4 \mathrm{~mm}$ ).

In conclusion, to the best of our knowledge, this is the first study that evaluated A. muciniphila for cell surface properties and some other features considered important for the screening of probiotic bacteria. For some characteristics, such as auto-aggregation, co-aggregation, hydrophobicity (toluene), and antimicrobial activity, the results obtained for A. muciniphila DSM 22959 were comparable with those of $L b$. rhamnosus GG.

The results related to sensitivity to antibiotics revealed a higher resistance of A. muciniphila DSM 22959 then that showed by $L b$. rhamnosus GG. This latter aspect certainly deserves a specific study, with particular attention to the presence of potentially transferable resistance genes.

Furthermore, interesting results derived from the positive effect of $L b$. rhamnosus GG on the growth of A. muciniphila when co-cultured.

Regarding the possible future use of $A$. muciniphila as a probiotic, it should be pointed out that there are no published human clinical trials that have studied the effects of $A$. muciniphila intake in humans, leading to a lack of conclusive evidence on its safety, and also the results obtained in this study are not conclusive on this point.

However, we agree with Brodmann et al. [54], who raise the question of whether it is necessary to prove the safety of an organism that is already part of nutrition in the early stages of life [14] and which constitutes $3 \%$ to $5 \%$ of the gut microbiota $[1,2]$.

Further insights are needed on this topic. The guidelines on probiotics as well as the European legislation should take specific cases such as A. muciniphila into account, as suggested by Brodmann et al. [54].

Author Contributions: A.C. and F.V.: Analysis and interpretation of the microbial data; drafting the work and revising it critically. P.T., M.I., S.J.L. and D.L.: Involved in experimental designing and data analysis. E.S.: Interpretation of data; drafting the work and revising it critically; responsibility of the accuracy or integrity of any part of the work. R.C., R.D.M. and M.S.: Conception of the work; experimental designing, drafting the work and revising it critically; responsibility for all aspects of the work in ensuring that questions related to the accuracy or integrity of any part of the work are appropriately investigated and resolved. All authors have read and agreed to the published version of the manuscript.

Funding: This research received no external funding.

Conflicts of Interest: The authors declare no conflict of interest.

\section{References}

1. Everard, A.; Belzer, C.; Geurts, L.; Ouwerkerk, J.P.; Druart, C.; Bindels, L.B.; Guiot, Y.; Derrien, M.; Muccioli, G.G.; Delzenne, N.M.; et al. Cross-talk between Akkermansia muciniphila and intestinal epithelium controls diet-induced obesity. Proc. Natl. Acad. Sci. USA 2013, 110, 9066-9071. [CrossRef]

2. Geerlings, S.; Kostopoulos, I.; de Vos, W.; Belzer, C. Akkermansia muciniphila in the Human Gastrointestinal Tract: When. Where, and How? Microorganisms 2018, 6, 75. [CrossRef] [PubMed] 
3. Derrien, M.; Vaughan, E.E.; Plunge, C.M.; de Vos, W.M. Akkermansia muciniphila gen. nov., sp. nov., a human intestinal mucin-degrading bacterium. Int. J. Sys. Evol. Microbiol 2004, 54, 1469-1476. [CrossRef] [PubMed]

4. Corazziari, E.S. Intestinal mucus barrier in normal and inflamed colon. J. Pediatr. Gastroenterol. Nutr. 2009, 48, S54-S55. [CrossRef] [PubMed]

5. Belzer, C.; de Vos, W.M. Microbes inside—from diversity to function: The case of Akkermansia. ISME J. 2012, 6, 1449-1458. [CrossRef] [PubMed]

6. Derrien, M.; Belzer, C.; de Vos, W.M. Akkermansia muciniphila and its role in regulating host functions. Microb. Pathog. 2017, 106, 171-181. [CrossRef]

7. Van Passel, M.W.J.; Kant, R.; Zoetendal, E.G.; Plugge, C.M.; Derrien, M.; Malfatti, S.A.; Chain, P.S.G.; Woyke, T.; Palva, A.; de Vos, W.M.; et al. The genome of Akkermansia muciniphila, a dedicated intestinal mucin degrader, and its use in exploring intestinal metagenomes. PLoS ONE 2011, 6, e16876. [CrossRef]

8. Derrien, M.; Van Baarlen, P.; Hooiveld, G.; Norin, E.; Muller, M.; De Vos, W. Modulation of mucosal immune response, tolerance, and proliferation in mice colonized by the mucin-degrader Akkermansia muciniphila. Front. Microbiol. 2011, 2, 166. [CrossRef]

9. Ottman, N.; Geerlings, S.Y.; Aalvink, S.; de Vos, W.M.; Belzer, C. Action and function of Akkermansia muciniphila in microbiome ecology, health and disease. Best Pract. Res. Clin. Gastroenterol. 2017, 31, 637-642. [CrossRef]

10. Weir, T.L.; Manter, D.K.; Sheflin, A.M.; Barnett, B.A.; Heuberger, A.L.; Ryan, E.P. Stool microbiome and metabolome differences between colorectal cancer patients and healthy adults. PLOS ONE 2013, 8, e70803. [CrossRef]

11. Zhang, T.; Li, Q.; Cheng, L.; Buch, H.; Zhang, F. Akkermansia muciniphila is a promising probiotic. Microb. Biotechnol. 2019, 2, 1109-1125. [CrossRef]

12. Cani, P.D.; de Vos, W.M. Next-Generation Beneficial Microbes: The Case of Akkermansia muciniphila. Front. Microbiol. 2017, 8, 1765. [CrossRef]

13. Gómez-Gallego, C.; Pohl, S.; Salminen, S.; De Vos, W.M.; Kneifel, W. Akkermansia muciniphila: A novel functional microbe with probiotic properties. Benef. Microbes 2016, 7, 571-584. [CrossRef]

14. Collado, M.C.; Laitinen, K.; Salminen, S.; Isolauri, E. Maternal weight and excessive weight gain during pregnancy modify the immunomodulatory potential of breast milk. Pediatr. Res. 2012, 72, 77-85. [CrossRef]

15. EFSA. Update of the list of QPS-recommended biological agents intentionally added to food or feed as notified to EFSA 8: Suitability of taxonomic units notified to EFSA until March 2018. EFSA J. 2018, 6, 531.

16. FAO/WHO. Guidelines for the Evaluation of Probiotics in Food. Available online: ftp://ftp.fao.org/es/esn/ food/wgreport2.pdf (accessed on 29 January 2020).

17. Succi, M.; Tremonte, P.; Pannella, G.; Tipaldi, L.; Cozzolino, A.; Romaniello, R.; Sorrentino, E.; Coppola, R. Pre-cultivation with selected prebiotics enhances the survival and the stress response of Lactobacillus rhamnosus in simulated gastrointestinal transit. Front. Microb. 2017, 8, 1067. [CrossRef]

18. Gorbach, S.; Doron, S.; Magro, F. Lactobacillus rhamnosus GG. The microbiota in gastrointestinal pathophysiology: implications for human health, prebiotics, probiotics, and dysbiosis; Floch, M.H., Ringel, Y., Walker, W.A., Eds.; Academic Press: Salt Lake City, UT, USA, 2017; chapter 7; pp. 79-88.

19. Valík, L.; Medved'ová, A.; Liptáková, D. Characterization of the growth Lactobacillus rhamnosus GG in milk at suboptimal temperature. J. Food Nutr. Res. 2008, 47, 60-67.

20. Succi, M.; Sorrentino, E.; Di Renzo, T.; Tremonte, P.; Reale, A.; Tipaldi, L.; Pannella, G.; Russo, A.; Coppola, R. Lactic acid bacteria in pharmaceutical formulations: presence and viability of "healthy microorganisms". J. Pharm. Nutr. 2014, 4, 66-75.

21. Succi, M.; Tremonte, P.; Reale, A.; Sorrentino, E.; Coppola, R. Preservation by freezing of potentially probiotic strains of Lactobacillus rhamnosus. Ann. Microbiol. 2007, 57, 537-544. [CrossRef]

22. EFSA. Guidance on the assessment of bacterial antimicrobial susceptibility. EFSA J. 2012, 10, 2740.

23. Klare, I.; Konstabel, C.; Müller-Bertling, S.; Reissbrodt, R.; Huys, G.; Vancanneyt, M.; Swings, J.; Goossens, J.H.; Witte, W. Evaluation of new broth media for microdilution antibiotic susceptibility testing of Lactobacilli, Pediococci, Lactococci, and Bifidobacteria. Appl. Environ. Microbiol. 2005, 71, 8982-8986. [CrossRef]

24. Collado, M.C.; Meriluoto, J.; Salminen, S. Adhesion and aggregation proprieties of probiotic and pathogen strain. Eur. Food Res. Technol. 2008, 226, 1065-1073. [CrossRef]

25. Del Re, B.; Sgorbati, B.; Miglioli, M.; Palenzona, D. Adhesion, auto-aggregation and hydrophobicity of 13 strains of Bifidobacterium longum. Lett. Appl. Microbiol. 2000, 31, 438-442. [CrossRef] 
26. Stepanovic, S.; Vukovic, D.; Dakic, I.; Savic, B.; Svabic-Vlahovic, M. A modified microtiter-plate test for quantification of staphylococcal biofilm formation. J. Microbiol. Methods 2000, 40, 175-179. [CrossRef]

27. Ruiz, L.; Sánchez, B.; de los Reyes-Gavilán, C.G.; Gueimonde, M.; Margolles, A. Coculture of Bifidobacterium longum and Bifidobacterium breve alters their protein expression profiles and enzymatic activities. Int. J. Food Microbiol. 2009, 133, 148-153. [CrossRef]

28. Kos, B.V.Z.E.; Šušković, J.; Vuković, S.; Šimpraga, M.; Frece, J.; Matošić, S. Adhesion and aggregation ability of probiotic strain Lactobacillus acidophilus M92. J. Appl. Microbiol. 2003, 94, 981-987. [CrossRef]

29. Tremonte, P.; Sorrentino, E.; Succi, M.; Tipaldi, L.; Pannella, G.; Ibañez, E.; Mendiola, J.A.; Di Renzo, T.; Reale, A.; Coppola, R. Antimicrobial effect of Malpighia punicifolia and extension of water buffalo steak shelf-life. J. Food Sci. 2016, 81, M97-M105. [CrossRef]

30. Tremonte, P.; Pannella, G.; Succi, M.; Tipaldi, L.; Sturchio, M.; Coppola, R.; Luongo, D.; Sorrentino, E. Antimicrobial activity of Lactobacillus plantarum strains isolated from different environments: A preliminary study. Int. Food Res. J. 2017, 24, 852-859.

31. Succi, M.; Tremonte, P.; Reale, A.; Sorrentino, E.; Grazia, L.; Pacifico, S.; Coppola, R. Bile salt and acid tolerance of Lactobacillus rhamnosus strains isolated from Parmigiano Reggiano cheese. FEMS Microbiol. Lett. 2005, 244, 129-137. [CrossRef]

32. Segers, M.E.; Lebeer, S. Towards a better understanding of Lactobacillus rhamnosus GG - host interactions. Microb. Cell Fact. 2014, 13, S7. [CrossRef]

33. Canani, B.R.; Nocerino, R.; Terrin, G.; Coruzzo, A.; Cosenza, L.; Leone, L.; Troncone, R. Effect of Lactobacillus GG on tolerance acquisition in infants with cow's milk allergy: a randomized trial. J. Allergy Clin. Immunol. 2012, 129, 580-582. [CrossRef] [PubMed]

34. Fong, F.L.Y.; Kirjavainen, P.; Wong, V.H.Y.; El-Nezami, H. Immunomodulatory effects of Lactobacillus rhamnosus GG on dendritic cells, macrophages and monocytes from healthy donors. J. Funct. Foods 2015, 13, 71-79. [CrossRef]

35. Coppola, R.; Succi, M.; Tremonte, P.; Reale, A.; Salzano, G.; Sorrentino, E. Antibiotic susceptibility of Lactobacillus rhamnosus strains isolated from Parmigiano Reggiano cheese. Le Lait 2005, 85, 193-204. [CrossRef]

36. Giri, S.S.; Sen, S.S.; Saha, S.; Sukumaran, V.; Park, S.C. Use of a potential probiotic, Lactobacillus plantarum L7, for the preparation of a rice-based fermented beverage. Front. Microbiol. 2018, 9, 473. [CrossRef]

37. Dubourg, G.; Lagier, J.C.; Armougom, F.; Robert, C.; Audoly, G.; Papazian, L.; Raoult, D. High-level colonisation of the human gut by Verrucomicrobia following broad-spectrum antibiotic treatment. Int. J. Antimicrob. Agents 2013, 41, 149-155. [CrossRef]

38. Guo, X.; Li, S.; Zhang, J.; Wu, F.; Li, X.; Wu, D.; Zhang, M.; Ou, Z.; Jie, Z.; Yan, Q.; et al. Genome sequencing of 39 Akkermansia muciniphila isolates reveals its population structure, genomic and functional diversity, and global distribution in mammalian gut microbiotas. BMC Genomics 2017, 18, 800. [CrossRef]

39. Santarmaki, V.; Kourkoutas, Y.; Zoumpopoulou, G.; Mavrogonatou, E.; Kiortzidis, M.; Chorianopoulos, N.; Tassou, C.; Tsakalidou, E.; Simopoulos, C.; Ypsilantis, P. Survival, Intestinal Mucosa Adhesion, and Immunomodulatory Potential of Lactobacillus plantarum Strains. Curr. Microb. 2017, 74, 1061-1067. [CrossRef]

40. Hory, K.; Matsumoto, S. Bacterial adhesion: From mechanism to control. Biochem. Eng. J. 2010, 48, 424-434. [CrossRef]

41. Duary, R.K.; Rajput, Y.S.; Batish, V.K.; Grover, S. Assessing the adhesion of putative indigenous probiotic lactobacilli to human colonic epithelial cells. Indian J. Med. Res. 2011, 134, 664-671.

42. Botes, M.; Loos, B.; van Reenen, C.A.; Dicks, L.M. Adhesion of the probiotic strains Enterococcus mundtii ST4SA and Lactobacillus plantarum 423 to Caco-2 cells under conditions simulating the intestinal tract, and in the presence of antibiotics and anti-inflammatory medicaments. Arch. Microbiol. 2008, 190, 573-584. [CrossRef]

43. Abdulla, A.A.; Abed, T.A.; Saeed, A.M. Adhesion, auto-aggregation and hydrophobicity of six Lactobacillus strains. Br. Microb. Res. J. 2014, 4, 381-391. [CrossRef]

44. Tuo, Y.; Yu, H.; Ai, L.; Wu, Z.; Guo, B.; Chen, W. Aggregation and adhesion properties of 22 Lactobacillus strains. J. Dairy Sci. 2013, 96, 4252-4257. [CrossRef]

45. Xu, H.; Jeong, H.S.; Lee, H.Y.; Ahn, J. Assessment of cell surface properties and adhesion potential of selected probiotic strains. Lett. Appl. Microbiol. 2009, 49, 434-442. [CrossRef]

46. Ekmekci, H.; Aslim, B.; Ozturk, S. Characterization of vaginal lactobacilli co-aggregation ability with Escherichia coli. Microbiol. Immunol. 2009, 53, 59-65. [CrossRef] 
47. Satyanarayana, T.; Johri, B.N.; Das, S.K. (Eds.) Microbial Diversity in Ecosystem Sustainability and Biotechnological Applications: Volume 1. Microbial Diversity in Normal E Extreme Environments; Springer: Singapore, 2019.

48. Gómez, N.C.; Ramiro, J.M.P.; Quecan, B.X.V.; de Melo Franco, B.D.G. Use of Potential Probiotic Lactic Acid Bacteria (LAB) Biofilms for the Control of Listeria monocytogenes, Salmonella Typhimurium, and Escherichia coli O157:H7 Biofilms Formation. Front. Microbiol. 2016, 7, 863. [CrossRef]

49. Pithva, S.; Shekh, S.; Dave, J.; Vyas, B.R.M. Probiotic attributes of autochthonous Lactobacillus rhamnosus strains of human origin. Appl. Biochem. Biotechnol. 2014, 173, 259-277. [CrossRef]

50. Lebeer, S.; Verhoeven, T.L.; Vélez, M.P.; Vanderleyden, J.; De Keersmaecker, S.C. Impact of environmental and genetic factors on biofilm formation by the probiotic strain Lactobacillus rhamnosus GG. Appl. Environ. Microbiol. 2007, 73, 6768-6775. [CrossRef]

51. Sánchez, B.; Burns, P.; Ruiz, L.; Binetti, A.; Vinderola, G.; Reinheimer, J.; Margolles, A.; Ruas-Madiedo, P.; de los Reyes-Gavilàn, C.G. Co-culture affects protein profile and heat tolerance of Lactobacillus delbrueckii subsp. lactis and Bifidobacterium longum. Food Res. Int. 2013, 54, 1080-1083.

52. Ferreira, C.L.B.; Grzeskowiak, L.B.; Collado, M.C.; Salminen, S. In vitro evaluation of Lactobacillus gasseri strains of infant origin on adhesion and aggregation of specific pathogens. J. Food Prot. 2011, 74, 1482-1487. [CrossRef]

53. Vlková, E.; Rada, V.; Šmehilová, M.; Killer, J. Auto-aggregation and co-aggregation ability in bifidobacteria and clostridia. Folia Microbiol. 2008, 53, 263-269. [CrossRef]

54. Brodmann, T.; Endo, A.; Gueimonde, M.; Vinderola, G.; Kneifel, W.; de Vos, W.M.; Gómez-Gallego, C. Safety of novel microbes for human consumption: practical examples of assessment in the European Union. Front. Microbiol. 2017, 8, 1725. [CrossRef]

(C) 2020 by the authors. Licensee MDPI, Basel, Switzerland. This article is an open access article distributed under the terms and conditions of the Creative Commons Attribution (CC BY) license (http://creativecommons.org/licenses/by/4.0/). 